\title{
Antagonistic cooperativity between antimalarials controlling hematin crystallization by attenuation of step pinning
}

\author{
WENCHUAN MA ${ }^{1}$, JAMES F. LUTSKO ${ }^{2}$, JEFFREY D. \\ RIMER $^{1}$, PETER G. VEKILOV ${ }^{1,3}$ \\ ${ }^{1}$ Department of Chemical and Biomolecular Engineering, \\ University of Houston, 4726 Calhoun Road, Houston, TX \\ 77204-4004, USA, vekilov@uh.edu \\ ${ }^{2}$ Center for Nonlinear Phenomena and Complex Systems, CP \\ 231, University Libre de Bruxelles, Boulevard du \\ Triomphe, 1050 Bruxelles, Belgium \\ ${ }^{3}$ Department of Chemistry, University of Houston, 3585 \\ Cullen Blvd., Houston, TX 77204-5003, USA
}

The pathology pathways of malaria, gout, kidney stones, and other aggregation diseases incorporate crystallization and its inhibition is the target of drugs that are often combined for increased efficacy. Hematin is a toxic byproduct of hemoglobin digestion in malaria parasites and suppression of its sequestration into inert hemozoin crystals is a favored approach to parasite elimination. Two or more hematin crystallization blockers have been combined in state-of-theart antimalarial regimens and extensive efforts have failed to deliver molecular-level understanding of the synergy or antagonism between the partner drugs. Here we demonstrate that drug pairs, whose constituents employ distinct mechanisms of hematin crystallization inhibition, kink blocking and a step pinning, exhibit both synergistic and antagonistic cooperativity depending on the drug combination and applied concentrations. Whereas synergism between two crystal growth modifiers is expected, the antagonistic cooperativity defies current crystal growth models. We demonstrate that the kink blockers reduce the line tension of the step edge, which facilitates both the nucleation of new crystal layers and the propagation of the steps through the gates created by step-pinners. The molecular viewpoint on synergistic and antagonist cooperativity between crystallization modifiers provides guidance on the pairing of drugs in malaria combination therapies and the understanding and control of physiological and pathological crystallization. In a broader context, our results highlight the complexity of crystal-modifier interactions mediated by the structures and dynamics of the crystal interface. 